Fatigue in Early Rheumatoid Arthritis: Data from the Early Rheumatoid Arthritis Network

Onosi Sylvia Ifesemen,1,2, Daniel Frederick McWilliams ${ }^{1,2,7}$, Sam Norton ${ }^{4}$, Patrick DW Kiely ${ }^{3}$, Adam

Young ${ }^{5}$, David Andrew Walsh ${ }^{1,2,6,7}$

1. Academic Rheumatology, University of Nottingham, Nottingham, United Kingdom

2. Pain Centre Versus Arthritis, University of Nottingham, Nottingham, United Kingdom

3. Department of Rheumatology, St Georges Hospital London

4. Kings College, London, United Kingdom

5. University of Hertfordshire, London, United Kingdom

6. Sherwood Forest NHS Foundation Trust, Nottinghamshire, United Kingdom

7. NIHR Nottingham Biomedical Research Centre, Nottingham, United Kingdom

Corresponding author

Onosi Ifesemen

Postal Address: Academic Rheumatology, University of Nottingham Clinical Sciences Building, City Hospital, Hucknall Road, Nottingham, United Kingdom, NG5 1PB

Email address: mbxosi@nottingham.ac.uk

ORCID : 0000-0002-4234-7905

Other authors ORCID reference numbers. DFM- 0000-0002-0581-1895; PK - 0000-0002-8091-2717, AY-0000-0001-5632-9807, SN-0000-0003-1714-9963

(C) The Author(s) 2021. Published by Oxford University Press on behalf of the British Society for Rheumatology. This is an Open Access article distributed under the terms of the Creative Commons Attribution Non-Commercial License (http://creativecommons.org/licenses/by-nc/4.0/), which permits non-commercial re-use, distribution, and reproduction in any medium, provided the original work is properly cited. For commercial re-use, please contact journals.permissions@oup.com 


\section{Fatigue in Early Rheumatoid Arthritis: Data from the Early Rheumatoid Arthritis Network.}

\section{ABSTRACT}

Objectives

Fatigue is a disabling symptom in people with Rheumatoid Arthritis (RA). This study aims to describe the prevalence, risk factors and the longitudinal course of fatigue in early RA.

Methods

Demographic, clinical, quality of life (QoL), comorbidities and laboratory data were from the Early Rheumatoid Arthritis Network (ERAN), a UK multicentre inception cohort of people with RA

Fatigue was measured using the Vitality subscale of SF36 where higher values represented better QoL. Baseline prevalences of fatigue classifications were age and sex standardised. Linear regression, hierarchical growth curve modelling and group-based trajectory modelling (GBTM) were utilized.

Results

At baseline ( $n=1236,67 \%$ female, mean age 57$)$, mean Vitality was 41 (SD \pm 11$)$, disease duration 11 months (IQR:7-18). Age and sex standardized prevalence rates of fatigue and severe fatigue were $44 \%$ (Cl: 39-50) and 19\% (Cl: 15-23) respectively.

Fatigue changed little over 3 years and 5 measurement occasions, $ß=-0.13(-0.23$ to -0.02$)$. GBTM identified 2 sub-groups, which we named 'Fatigue' (53\%) and 'No-fatigue' (47\%) groups. Female sex, worse pain, mental health, and functional ability were associated with greater fatigue and predicted 'Fatigue' group membership (AUROC=0.81). Objective measures of inflammation - swollen joint count (SJC) and erythrocyte sedimentation rate (ESR) were not significantly associated with fatigue.

Conclusions

Fatigue is prevalent and persistent in early RA. Diverse characteristics indicative of central mechanisms are associated with persistent fatigue. Management of fatigue might require interventions targeted at central mechanisms in addition to inflammatory disease modification. People who require such interventions might be identified at presentation with early RA.

Keywords: Fatigue, Rheumatoid Arthritis, Trajectories, Inflammation, Central mechanisms 


\section{Key Messages}

1. Fatigue is prevalent and remains stable regardless of improvements in inflammatory disease activity.

2. People with persistent fatigue could be identified early, representing targets for fatigue lowering interventions.

3. Traits indicative of central mechanisms are associated with persistent fatigue in early RA.

\section{Introduction}

Fatigue is a common debilitating symptom in many musculoskeletal diseases. In rheumatoid arthritis (RA), the prevalence of fatigue has been reported to be between $40-70 \%$ (1-3). This large variation stems from a heterogenous RA population, at various levels of disease activity, and from the use of different fatigue measurement tools. Fatigue is associated with greater healthcare utilization and worse outcomes. Fatigue is also associated with huge economic consequences, being responsible for sickness absence and loss of employment, culminating in an overall poorer quality of life in these individuals(4).

Fatigue was recommended as a core outcome measure in RA clinical trials and a key symptom whose absence is required to indicate RA remission from the patient's perspective over a decade ago, however in spite of the resulting increase in RA fatigue research, the exact causal mechanisms remain elusive(5). Fatigue was traditionally thought to be a consequence of an inflammatory process; however, despite the innovations in anti-inflammatory therapeutics, fatigue remains a problem for many people with $\mathrm{RA}(6)$.

Several conceptual causal models have been proposed, incorporating biopsychosocial and environmental factors, as direct and cumulative causal effects. Supporting evidence is largely based on cross-sectional studies, and studies involving individuals with longstanding disease(6, 7). Few longitudinal studies describe the progression of fatigue over time, and a large proportion of these studies include people with long standing disease, and disease refractory to first line anti rheumatic therapy (8). Fatigue in RA has been characterised as persistent and stable over time, or to improve in some participant subgroups, but persist in others. Generalisation from these study findings is limited by small sample sizes and heterogeneity between study populations and contexts (8-10).

Several studies have shown the benefit of early treatment of RA on disease activity. Clinical trials demonstrated a small reduction in fatigue levels with the use of biologic disease modifying antirheumatic drugs (DMARDs), although the extent to which these findings can be generalised to other DMARDs, or to early RA is unknown(11). This study aims to characterise the prevalence and course of fatigue and, to identify characteristics associated or predictive of fatigue in people with early rheumatoid arthritis We hypothesized that groups of individuals may exist who exhibit varying fatigue 
progression and that identification of these discrete trajectory groups might already be possible in individuals with early disease, based on demographic or clinical characteristics.

\section{Methods}

\section{Data sources}

Data from the early rheumatoid arthritis network (ERAN) were analysed. ERAN has been described in detail elsewhere (12). In brief, people with a symptom duration of less than 2 years were recruited on clinician diagnosis of rheumatoid arthritis from outpatient clinics in the UK and Eire between 2002 2012. Participants were excluded from the cohort if found to have an alternative diagnosis at follow up and were not analysed in this study. If participants did not attend study visits at their centre for any reason, they became lost to follow up. Demographic, clinical, and laboratory data were collected at baseline, 6 months and annually thereafter. ERAN contains a wealth information on early RA and has been used in several published articles on the epidemiology of early Rheumatoid Arthritis and in the development of National Institute of Health and Care Excellence (NICE) decision rules(13). Data from the first 5 assessment visits, equivalent to 3 years of follow up were used for longitudinal analyses in this study. Ethical approval was from the UK National Health Service (Trent Research Ethics Committee reference 01/4/047). All participants gave written informed consent, and the study was conducted in accordance with the declaration of Helsinki. This study was reported in accordance with the Strengthening the Reporting of Observational Studies in Epidemiology (STROBE) guidelines for cohort studies (Supplementary Table S1, available at Rheumatology online).

\section{Measures \\ Outcome}

Fatigue was measured using the vitality subscale of the Medical Outcomes Study Short-Form 36 (Vitality)(14). In accordance with the developer's scoring guidelines, UK population normalised values were utilised resulting in a $0-100$ scale, with higher scores signifying a better quality of life (QoL). Vitality has good psychometric properties and is often used to assess fatigue in musculoskeletal research(14).

A binary $(y / n)$ fatigue variable was derived from Vitality to estimate fatigue prevalence. The crude prevalence of fatigue and severe fatigue were estimated as the proportion of participants with Vitality $\leq 1 \mathrm{SD}$ and $2 \mathrm{SD}$ of the UK population mean score of 50 respectively (15). 1 SD of UK normalised values corresponds to the minimum clinically important difference (MCID) of SF-36 vitality(16). Prevalence values standardised to the European standard population (ESP) 2013 were estimated using the $R$ statistical software 'Epitools' package(17). ESP is an artificial population structure based on the population structures of European countries. It is used to estimate age and sex standardised rates. 


\section{Exposures}

Demographic characteristics - self-reported age at onset of disease (in years), sex (male/female), and ethnicity (white/non-white), body mass index $\left(\mathrm{kg} / \mathrm{m}^{2}\right)$, smoking status (never, current and ex) were included in the analysis.

Inflammation/ Disease Activity was measured using Erythrocyte Sedimentation Rate (ESR) in mm/hr. Tender (TJC) and swollen joint counts (SJC) in 28 joints, each scaled $0-28$, and Patient's Global Assessment of Disease Activity (PGA) on a $0-100 \mathrm{~mm}$ visual analogue scale(18). In addition disease activity score in 28 joints (DAS28), a composite index of disease activity comprising TJC, SJC, PGA and ESR were assessed(19). Seropositivity was classified according to the presence of rheumatoid factor and/or positive or borderline anti-cyclic citrullinated peptide antibodies (CCP)(20). The presence or absence of nodules on clinical examination were also assessed.

Comorbidities were assessed using the Rheumatic Disease Comorbidity Index (RDCI)(21).

Pain and Mental Health were measured with SF36 bodily pain (SF36BP) and SF36 mental health (SF36MH) subscales, respectively. Like Vitality, these subscales were normalised to the UK population on a $0-100$ scale with higher values representing a better QoL. SF36MH assesses mental symptoms and psychological wellbeing $(22,23)$.

Disability/Functional Limitation was measured using the Health Assessment Questionnaire (HAQ) Disability Index (HAQ-DI). This questionnaire assesses the level of functional ability by examining functional activities such as walking, eating, reach, grip, and usual activities. It is scored on a scale of $0-3$, with higher scores indicating greater disability(24).

Medication use was represented by a binary $(\mathrm{y} / \mathrm{n})$ variable, with the affirmative comprising participants prescribed disease modifying anti-rheumatic drugs (DMARDs).

Disease duration was measured by the self-reported time since onset of symptoms in months.

Time was assessed as the study measurement occasion. The first measurement occasion was defined as that at baseline, and the second was at 3 to 6 months. Subsequent measurements were conducted annually from baseline. Data were used up to the $5^{\text {th }}$ measurement occasion ( 3 years from baseline).

\section{Statistical Analysis}

The overall study sample comprised data from the first 5 measurement occasions. Data for subsequent measurement occasions were excluded due to high attrition rates. Sample data were summarised using descriptive statistics at baseline and at each measurement occasion.

Factors associated with baseline Vitality scores were examined using Spearman's correlation coefficients whose magnitudes were interpreted according to published guidelines(25). Baseline Vitality scores were also examined using a univariate and multiple linear regression, with robust 
standard errors. Multicollinearity was tested using variance inflation factor (VIF). Residuals were examined graphically to assess linear regression assumptions.

Individuals with 3 or more Vitality measurements were included in the longitudinal analysis using hierarchical growth curve analysis. Models with random intercepts for person and a random slope for person and time were used to capture between person variability over time(26). Linear, quadratic, and linear spline trajectories were investigated. Following trajectory selection, the unconditional (model without covariates) and conditional (with covariates) models were examined. The model that best suited the data based on the Bayesian Information Criteria (BIC), log likelihood and Akaike Information Criteria (AIC) was selected (27). Missing data were considered missing at random (MAR) and handled using multiple imputation using chained equations, accounting for the longitudinal structure of the data. 100 imputations using 10 burn-in iterations were conducted using the 'miceadds' R package $(28,29)$.

Fatigue trajectories were investigated using Group Based Trajectory Modelling (GBTM). GBTM is an application of finite mixture models that gathers individuals into subgroups with similar growth trajectories based on the estimated probability of group membership. Model fit was assessed using AIC, BIC, log-likelihood (LL) and Entropy. Entropy indicates how well groups are separated and how well individuals fit into their respective groups(30). Resultant group assignment was assessed using posterior probabilities and odds of correct classification. Posterior probabilities are the probabilities of group membership derived from parameter estimates of the model. An average posterior probability of $>0.7$ and odds of correct classification $>5$ for each group was deemed acceptable(31). GBTM was conducted using the STATA ${ }^{\circledR}$ 'Traj' plugin(31).

Baseline risk factors for fatigue group membership were investigated using univariate and multivariable logistic regression analysis. Model discrimination was examined using the Area Under the Receiver Operating Characteristic (ROC) Curve.

Sensitivity analyses were conducted by restricting the study population to participants who had a selfreported disease duration of less than 6 months and restricting longitudinal analysis to participants with 5 complete vitality measurements.

Analyses were performed using STATA 16 , and R. P values $\leq 0.05$ were considered statistically significant unless otherwise stated.

\section{Results}

The ERAN sample at baseline comprised 1236 participants, of whom 992 had Vitality scores recorded at baseline. Three or more Vitality scores were recorded by the $5^{\text {th }}$ measurement occasion for 792 participants, with Vitality scores for 493 individuals in year 3 (Supplementary Figure S1, available at Rheumatology online). 
Table 1 describes the study sample at baseline. The sample was predominantly female (67\%) of white ethnicity (96\%), with a mean (sd) age of 57 years $( \pm 14)$ and mean Vitality score of $41( \pm 11)$. $61 \%$ of participants were seropositive. Mean Pain and Mental Health scores at baseline were $33( \pm 10)$ and 42 $( \pm 11)$ respectively, and median disease duration was 11 months (IQR $7-18)$. Mean baseline DAS28ESR was $4.7( \pm 1.6)$. Descriptive characteristic of the ERAN population at each measurement occasion showed an improvement in disease activity/inflammatory characteristics (TJC, SJC, PGA, DASESR) and HAQ (Supplementary Table S2, available at Rheumatology online).

\section{Prevalence and associations of Fatigue at Baseline}

Over $75 \%$ of the study population reported lower Vitality scores than the mean UK general population value of 50. Age and sex standardized prevalence of fatigue and severe fatigue at baseline were $44 \%$ $(\mathrm{Cl}: 39-50)$ and 19\% $(\mathrm{Cl}: 15-23)$ respectively. Similar prevalence values were seen in participants with less than 6 months disease duration (Supplementary Table S3, available at Rheumatology online).

Pain and Mental Health scores showed moderate positive correlation with Vitality scores at baseline $(r=0.54 \& 0.61$ respectively, $\mathrm{P} \leq 0.05)$. HAQ was negatively correlated with Vitality $(r=-0.50, P \leq$ 0.05). Associations between Vitality scores and other variables at baseline were weak (Supplementary Table S4, available at Rheumatology online).

Female sex, worse pain, mental health, TJC, SJC, PGA, Hb, SJC, RDCl, ESR, DAS28ESR and higher BMI were significantly associated with Vitality at baseline in the bivariate regression analysis (Supplementary Table S5, available at Rheumatology online). Table 2 describes multivariable linear regresion analysis; female sex, worse mental health, pain, HAQ and TJC were significantly associated with Vitality. Collectively, these characteristics explained about half of Vitality variability at baseline $\left(R^{2}=0.49\right)$. Mental health and pain showed the largest contibution to Vitality (standardized $\beta=0.42$ and 0.24 respectively). Multicollinearity was not detected (mean VIF $=1.47$, none $\geq 2$ ) and linear regression assumptions were not violated (Supplementary Figure S2A and B, available at Rheumatology online).

\section{Course of fatigue over time}

729 participants were included in the longitudinal analysis. These participants appeared similar to the total ERAN population in terms of measured covariates (Supplementary Table S6, available at Rheumatology online). Missing data proportions are provided in Supplementary Figure S3, available at Rheumatology online.

Mean Vitality remained relatively stable throughout the period of the study with little variability between measurement occasions (Figure 1 and Supplementary Figure S4, available at Rheumatology online). The linear growth model was selected as the best fit to the data based on fit statistics (Supplementary Table S7A and B, available at Rheumatology online). Estimates from the unconditional linear growth model (model without covariates), the mean Vitality score at baseline was $42.32(\mathrm{Cl}$ : 
41.58-43.06), with an increase in vitality over the study duration, of $\beta=0.19$ ( $\mathrm{Cl}: 0.05-0.33)$ at each measurement occasion. The fully conditional model showed little chanage over time, $\beta=-0.13(-0.23$ to -0.02$)$, and the slope of the Vitality trajectory was not affected by the presence of covariates (Supplementary Table S8, available at Rheumatology online).

\section{Group Based Trajectory Modelling (GBTM)}

GBTM analysis of Vitality scores revealed 2 trajectory groups, which we named the 'Fatigue' (47\%) and 'No-fatigue' (53\%) groups (Figure 2). The 2 trajectory group estimates had lower AIC, BIC, LL, and a higher entropy than a 3-trajectory group model (Supplementary Table S9, available at Rheumatology online). The average posterior probabilities and odds of correct classification were $94 \%$ and 13.7 for Fatigue; and 95\% and 21.19 for No-fatigue groups respectively (Supplementary Table S10, available at Rheumatology online). Participants in the No-fatigue group had mean Vitality scores of 49.67 (Cl: 48.70 - 50.64), while for those in the Fatigue group, mean vitality scores were $>1$ standard deviation less than the average population levels, 35.23 (Cl: 34.45 - 36.00) (Supplementary Table S11, available at Rheumatology online).

Table 3 describes baseline characteristics of the Vitality trajectory groups. Individuals in the Fatigue group were predominantly female, had higher DAS28, worse pain and mental health, and worse HAQ and PGA than those in the No-fatigue group. Table 4 shows the results of the univariate and multivariable regression analysis estimating the association between baseline characteristics and group membership. Female sex, higher BMI, higher $\mathrm{HAQ}$, worse mental health, worse pain, higher DAS28 and higher RDCl at baseline were all associated with membership of the Fatigue group. The Area under the Receiver operating characteristic curve (AUROC) was 0.81 (Supplementary Figure S5, available at Rheumatology online).

Sensitivity analysis showed similar results when data from participants who had a disease duration of less than 6 months at baseline, or from participants with 5 complete SF36 Vitality measurements were analysed (Supplementary Tables S12A to 13B, available at Rheumatology online). Analyses using unimputed data also provided similar results.

\section{Discussion}

Fatigue is prevalent in early RA and associated with worse patient reported outcomes even in early disease. Our study found that in early RA, over half of the RA population experienced clinically significant fatigue. Fatigue remained persistent and did not undergo clinically significant change over time. Females with worse mental health, pain, and functional ability at presentation with RA were more likely to experience fatigue throughout the course of disease. 
Our study aligns with previous studies, which reported similar high prevalence rates in established RA in both UK and non-UK populations $(1,2)$; highlighting the problem across the disease course and in different populations.

This study assessed the course of fatigue over the first three years or RA and overall, mean vitality scores were remarkably stable over the duration, with values less than the minimum clinically important difference (MCID) of Vitality (16), meaning that people with fatigue at baseline continued to report fatigue longitudinally. A deeper examination of the data using trajectory analyses found two distinct groups, with or without fatigue. This result reflects our findings on the prevalence of fatigue, as not all people experience clinically important fatigue in RA, however those who experience fatigue at baseline continued to report fatigue at follow up.

We identified that female sex, pain, mental health, and functional ability showed a consistent association with fatigue when examined cross sectionally and predicted people more likely to belong to the Fatigue group. This concurs with findings from other (32) and are mirrored in people with inflammatory and non-inflammatory diseases including post-viral fatigue and post-chemotherapy fatigue $(33,34)$. Interestingly, many RA-related characteristics, such as inflammation and duration of disease were not consistently significantly associated with fatigue cross sectionally and longitudinal in our analyses(35).These results corroborate findings from other cross-sectional studies that found little association between traditional measures of inflammation (ESR and CRP) and fatigue(36, 37). Although, some previous studies reported an association between inflammation and fatigue, these studies either used univariate analysis or a composite measure of disease activity e.g. DAS28 $(32,38)$. In addition, fatigue has been demonstrated to persist in the presence of well controlled inflammatory disease, and in conditions with no strong evidence of systemic inflammatory component (e.g. fibromyalgia) (39).

Female sex, worse mental health, pain, and functional capacity were consistently associated with fatigue in our study. These factors, and indeed fatigue are components of a cluster of characteristics described as fibromyalgianess. Fibromyalgianess is associated with central sensitization, involving hyper-excitement of the central neurons characterised by amplification of noxious stimulus and increased sensitivity to environmental stimuli such as heat or light (40). Central sensitization is an established pain mechanism in musculoskeletal diseases and evidence of an association with fatigue has been reported(41). Central sensitization could explain fatigue persistence and association with pain, metal health and lower functional capacity and overall worse outcomes even in the presence of well controlled inflammatory disease(42, 43).

It is noteworthy that randomised controlled trials (RCTs) showed a small improvement in fatigue levels after 6 months of follow up after treatment with biologic DMARD therapies, although these studies did not examine the long-term changes in fatigue(44). It remains possible, therefore that fatigue may originate from an inflammatory process, whereby proinflammatory cytokines fundamental to the 
development of an immune response trigger long term changes in brain architecture, neural pathways, and sensitisation culminating in central sensitization and fibromyalgianess(45).

RCTs have also demonstrated a therapeutic 'window of opportunity' in RA management associated with better outcomes in terms of radiological damage and disability (11). There is some debate about the duration of this therapeutic window, however, up to 2 years post-diagnosis is supported by the literature. It is proposed that intervening while the disease process is less mature and more reversible would facilitate the modulation of the disease. This non-linear progression has been demonstrated in studies describing disease activity, pain, and psychological distress in RA (12). Our study although not designed to examine the effect of treatment revealed that fatigue was present at the start of RA and underscores another consideration that the causes of fatigue may occur much earlier in the disease course, possibly even in preclinical stages of the disease. More studies in populations at risk of developing RA may provide greater insight into the mechanisms of fatigue(46).

Previous studies on the progression of fatigue in established RA identified additional groups, with improving fatigue, and worsening fatigue. In one of the studies, individuals were assigned to groups $a$ priori based on values of the fatigue measure on a population starting biologic DMARD therapy(8). The improving fatigue group could result from higher baseline fatigue in the study population and/or better control of inflammation with the initiation of biologic DMARD therapy. Another study found two persistent fatigue groups in women only, in contrast, our study did not elicit the effect of sex on trajectory groups, although females had lower starting fatigue than males in our study population. Overall, the pattern of persistent fatigue in certain groups of individuals with RA was consistent across all studies $(8,47)$.

Current RA treatment guidelines include therapeutic management, with DMARDs and access to a multidisciplinary team to manage other symptoms that affect the quality of life of in these individuals, including fatigue. There is little published data on the uptake of these services and paucity of information on specific interventions geared towards the management of symptoms in people with RA, suggesting that they are not used very frequently. This is probably due to the hope that improving control of inflammatory disease might resolve other problematic symptoms, and maybe also the challenges of identifying people with definite central sensitization. Additional tools to identify people with central mechanism traits in clinical practice are being developed and would provide a means to identify individuals that may require additional treatments over peripheral pain and inflammation(48, 49).

This study is subject to some limitations. High attrition rates precluded the inclusion of additional timepoints to the longitudinal analysis. However, GBTM produces unbiased results under the MAR assumption(30) and we did not detect an effect from attrition on our findings. Data were assumed to be missing at random. Results may be subject to bias if data were missing not at random, although sensitivity analyses using unimputed data provided similar findings. Cases were eligible for 
participation in ERAN if they had a physician diagnosis of RA, and subsequently excluded if clinical diagnosis was revised. However, it remains possible that some participants may have been inadvertently included with diagnoses other than RA.

RA treatment strategies have evolved since the inception of this cohort, and it is not clear if these new treatment strategies would present a different picture. This study did not address some notable factors associated with fatigue (e.g. sleep quality). Univariate GBTM was used, perhaps, modelling joint trajectories of other significant fatigue risk factors would provide more insight to the nature of heterogeneity observed in this analysis.

\section{Conclusion}

Fatigue is a prevalent symptom in RA, even in early disease. Those with fatigue at baseline were likely to continue to report fatigue at follow up. Diverse baseline characteristics, indicative of central mechanisms are associated with persistent fatigue. Management of fatigue might require complex interventions targeted at central mechanisms in addition to disease modification, and people who require such interventions might be identified at presentation with early RA.

\section{Authors Funding Statement}

This work is supported by the Versus Arthritis pain Centre Grant Number 20777 and Onosi Ifesemen's PhD is partially funded by the Early Rheumatoid Arthritis Network (ERAN)

\section{Disclosure Statement}

Daniel Fredrick McWilliams received grant support from Pfizer and Eli Lilly, David Andrew Walsh received grant support from Pfizer, Eli Lilly, UCB. Consultancy for Pfizer, GSK, AbbVie.

\section{Data availability Statement}

The data underlying this article will be shared on reasonable request to the corresponding author.

\section{References}

1. Wolfe F, Hawley DJ, Wilson K. The prevalence and meaning of fatigue in rheumatic disease. J Rheumatol. 1996;23(8):1407-17.

2. Overman CL, Kool MB, Da Silva JA, Geenen R. The prevalence of severe fatigue in rheumatic diseases: an international study. Clin Rheumatol. 2016;35(2):409-15.

3. Nikolaus S, Bode C, Taal E, van de Laar MA. Fatigue and factors related to fatigue in rheumatoid arthritis: a systematic review. Arthritis Care Res (Hoboken). 2013;65(7):1128-46.

4. Katz P. Fatigue in Rheumatoid Arthritis. Curr Rheumatol Rep. 2017;19(5):25. 
5. Kirwan J, Heiberg $T$, Hewlett $S$, Hughes R, Kvien $T$, Ahlmèn $M$, et al. Outcomes from the Patient Perspective Workshop at OMERACT 6. The Journal of rheumatology. 2003;30(4):868-72.

6. Hewlett S, Chalder T, Choy E, Cramp F, Davis B, Dures E, et al. Fatigue in rheumatoid arthritis: time for a conceptual model. Rheumatology. 2011;50(6):1004-6.

7. Geenen R, Dures E. A biopsychosocial network model of fatigue in rheumatoid arthritis: a systematic review. Rheumatology. 2019;58(Supplement_5):v10-v21.

8. Provan SA, Michelsen B, Sexton J, Uhlig T, Hammer HB. Trajectories of fatigue in actively treated patients with established rheumatoid arthritis starting biologic DMARD therapy. RMD Open. 2020;6(3):e001372.

9. Repping-Wuts H, Fransen J, van Achterberg T, Bleijenberg G, van Riel P. Persistent severe fatigue in patients with rheumatoid arthritis. J Clin Nurs. 2007;16(11c):377-83.

10. van Steenbergen HW, Tsonaka R, Huizinga TWJ, Boonen A, van der Helm-van Mil AHM. Fatigue in rheumatoid arthritis; a persistent problem: a large longitudinal study. RMD Open. 2015;1(1):e000041.

11. Chauffier K, Salliot C, Berenbaum F, Sellam J. Effect of biotherapies on fatigue in rheumatoid arthritis: a systematic review of the literature and meta-analysis. Rheumatology (Oxford). 2012;51(1):60-8.

12. McWilliams DF, Dawson O, Young A, Kiely PDW, Ferguson E, Walsh DA. Discrete Trajectories of Resolving and Persistent Pain in People With Rheumatoid Arthritis Despite Undergoing Treatment for Inflammation: Results From Three UK Cohorts. J Pain. 2019;20(6):716-27.

13. Laura Gibson MHA, Allan Wailoo. Progression of Disease in People with Rheumatoid Arthritis treated with Non Biologic Therapies - Report by the Decision Support Unit. United Kingdom: School of Health and Related Research, University of Sheffield 2015.

14. Hewlett S, Hehir M, Kirwan JR. Measuring fatigue in rheumatoid arthritis: a systematic review of scales in use. Arthritis Rheum. 2007;57(3):429-39.

15. van Hoogmoed D, Fransen J, Bleijenberg G, van Riel P. Physical and psychosocial correlates of severe fatigue in rheumatoid arthritis. Rheumatology (Oxford). 2010;49(7):1294-302.

16. Bjorner JB, Wallenstein GV, Martin MC, Lin P, Blaisdell-Gross B, Tak Piech C, et al. Interpreting score differences in the SF-36 Vitality scale: using clinical conditions and functional outcomes to define the minimally important difference. Current Medical Research and Opinion. 2007;23(4):731-9.

17. Tomas J. Aragon MPF, Daniel Wollschlaeger, Adam Omidpanah. Package 'epitools'. 0.5-10.1 ed2020. p. Tools for training and practicing epidemiologists including methods for twoway and multi-way contingency tables.

18. Nikiphorou E, Radner H, Chatzidionysiou K, Desthieux C, Zabalan C, van Eijk-Hustings $\mathrm{Y}$, et al. Patient global assessment in measuring disease activity in rheumatoid arthritis: a review of the literature. Arthritis Research \& Therapy. 2016;18(1):251.

19. Wells G, Becker JC, Teng J, Dougados M, Schiff M, Smolen J, et al. Validation of the 28-joint Disease Activity Score (DAS28) and European League Against Rheumatism response criteria based on C-reactive protein against disease progression in patients with rheumatoid arthritis, and comparison with the DAS28 based on erythrocyte sedimentation rate. Annals of the Rheumatic Diseases. 2009;68(6):954.

20. Lee AN, Beck CE, Hall M. Rheumatoid factor and anti-CCP autoantibodies in rheumatoid arthritis: a review. Clinical Laboratory Science. 2008;21(1):15.

21. England BR, Sayles H, Mikuls TR, Johnson DS, Michaud K. Validation of the rheumatic disease comorbidity index. Arthritis Care Res (Hoboken). 2015;67(6):865-72.

22. Hawker GA, Mian S, Kendzerska T, French M. Measures of adult pain: Visual analog scale for pain (vas pain), numeric rating scale for pain (nrs pain), mcgill pain questionnaire (mpq), short-form mcgill pain questionnaire (sf-mpq), chronic pain grade scale (cpgs), short form-36 bodily pain scale (sf-36 bps), and measure of intermittent and constant osteoarthritis pain (icoap). Arthritis care \& research. 2011;63(S11):S240-S52.

23. Bech P, Olsen LR, Kjoller M, Rasmussen NK. Measuring well-being rather than the absence of distress symptoms: a comparison of the SF-36 Mental Health subscale and the WHO-Five well-being scale. International Journal of Methods in Psychiatric Research. 2003;12(2):85-91.

24. Bruce B, Fries JF. The Stanford Health Assessment Questionnaire: Dimensions and Practical Applications. Health and Quality of Life Outcomes. 2003;1(1):20. 
25. Schober P, Boer C, Schwarte LA. Correlation Coefficients: Appropriate Use and Interpretation. Anesthesia \& Analgesia. 2018;126(5):1763-8.

26. Curran PJ, Obeidat K, Losardo D. Twelve Frequently Asked Questions About Growth Curve Modeling. J Cogn Dev. 2010;11(2):121-36.

27. Burnham KP, Anderson DR. Multimodel Inference: Understanding AIC and BIC in Model Selection. Sociological Methods \& Research. 2004;33(2):261-304.

28. Robitzsch A, Grund S, Henke T, Robitzsch MA. Package 'miceadds'. R Package: Madison, WI, USA. 2017.

29. Carpenter JR, Smuk M. Missing data: A statistical framework for practice. Biometrical Journal. 2021;63(5):915-47.

30. Nagin DS, Odgers CL. Group-based trajectory modeling in clinical research. Annu Rev Clin Psychol. 2010;6:109-38.

31. Daniel SN. Group-Based Trajectory Modeling: An Overview. Annals of nutrition and metabolism. 2014;65(2/3):205-10.

32. Rat A-C, Pouchot J, Fautrel B, Boumier P, Goupille P, Guillemin F. Factors associated with fatigue in early arthritis: Results from a multicenter national French cohort study. Arthritis Care \& Research. 2012;64(7):1061-9.

33. Esbensen BA, Stallknecht SE, Madsen ME, Hagelund L, Pilgaard T. Correlations of fatigue in Danish patients with rheumatoid arthritis, psoriatic arthritis and spondyloarthritis. PLoS One. 2020;15(8):e0237117.

34. Bearne LM, Bieles J, Georgopoulou S, Andrews J, Tully A, Stolarchuk-Prowting K, et al. Fatigue in adults with primary antiphospholipid syndrome: findings from a mixed-methods study. Lupus. 2020;29(8):924-33.

35. Minnock P, Veale DJ, Bresnihan B, FitzGerald O, McKee G. Factors that influence fatigue status in patients with severe rheumatoid arthritis (RA) and good disease outcome following 6 months of TNF inhibitor therapy: a comparative analysis. Clin Rheumatol. 2015;34(11):1857-65.

36. Stebbings S, Herbison P, Doyle TC, Treharne GJ, Highton J. A comparison of fatigue correlates in rheumatoid arthritis and osteoarthritis: disparity in associations with disability, anxiety and sleep disturbance. Rheumatology (Oxford). 2010;49(2):361-7.

37. Bergman MJ, Shahouri SH, Shaver TS, Anderson JD, Weidensaul DN, Busch RE, et al. Is fatigue an inflammatory variable in rheumatoid arthritis (RA)? Analyses of fatigue in RA, osteoarthritis, and fibromyalgia. J Rheumatol. 2009;36(12):2788-94.

38. Huyser BA, Parker JC, Thoreson R, Smarr KL, Johnson JC, Hoffman R. Predictors of subjective fatigue among individuals with rheumatoid arthritis. Arthritis \& Rheumatism. 1998;41(12):2230-7.

39. Druce KL, Bhattacharya Y, Jones GT, Macfarlane GJ, Basu N. Most patients who reach disease remission following anti-TNF therapy continue to report fatigue: results from the British Society for Rheumatology Biologics Register for Rheumatoid Arthritis. Rheumatology (Oxford).

2016;55(10):1786-90.

40. Yunus MB. Fibromyalgia and Overlapping Disorders: The Unifying Concept of Central Sensitivity Syndromes. Seminars in Arthritis and Rheumatism. 2007;36(6):339-56.

41. Druce KL, McBeth J. Central sensitization predicts greater fatigue independently of musculoskeletal pain. Rheumatology. 2019;58(11):1923-7.

42. Lee YC, Napadow V, Loggia ML. Editorial: Functional Connectivity: Dissecting the Relationship Between the Brain and "Pain Centralization" in Rheumatoid Arthritis. Arthritis Rheumatol. 2018;70(7):977-80.

43. Wolfe F. Fibromyalgianess. Arthritis Care \& Research. 2009;61(6):715-6.

44. Almeida C, Choy EH, Hewlett S, Kirwan JR, Cramp F, Chalder T, et al. Biologic interventions for fatigue in rheumatoid arthritis. Cochrane Database Syst Rev. 2016;2016(6):Cd008334.

45. Korte SM, Straub RH. Fatigue in inflammatory rheumatic disorders: pathophysiological mechanisms. Rheumatology (Oxford, England). 2019;58(Suppl 5):v35-v50.

46. Deane KD, Norris JM, Holers VM. Preclinical rheumatoid arthritis: identification, evaluation, and future directions for investigation. Rheum Dis Clin North Am. 2010;36(2):213-41.

47. Druce KL, Jones GT, Macfarlane GJ, Verstappen SM, Basu N. The Longitudinal Course of Fatigue in Rheumatoid Arthritis: Results from the Norfolk Arthritis Register. J Rheumatol.

2015;42(11):2059-65. 
48. Akin-Akinyosoye K, Frowd N, Marshall L, Stocks J, Fernandes GS, Valdes A, et al. Traits associated with central pain augmentation in the Knee Pain In the Community (KPIC) cohort. Pain. 2018;159(6):1035-44.

49. Ifesemen OS, McWilliams DF, Ferguson E, Wakefield R, Akin-Akinyosoye K, Wilson D, et al. Central Aspects of Pain in Rheumatoid Arthritis (CAP-RA): protocol for a prospective observational study. BMC Rheumatology. 2021;5(1):23.

Tables and figures

Table 1:Overview of ERAN Population at Baseline

\begin{tabular}{lc}
\hline Characteristics, unit & Values \\
\hline SF36VT & $41.82(11.14)$ \\
\hline Demographics & $57.01(14.03)$ \\
\hline Age, yrs. & $839(67.88)$ \\
\hline Female* & $1196(97.00)$ \\
\hline White ethnicity* & $26.84(16.80-29.76)$ \\
\hline BMI, Kg/M²(Median, IQR) & $477(40.02)$ \\
\hline Never Smoker* & $404(33.89)$ \\
\hline Ex-Smoker* & $311(26.09)$ \\
\hline Current Smoker* & \\
\hline Disease Activity/Inflammation & $4.68(1.56)$ \\
\hline DAS28ESR & $5.00(2-11)$ \\
\hline TJC (Median, IQR) & $4.00(1-9)$ \\
\hline SJC (Median, IQR) & $655(61.04)$ \\
\hline Seropositive* & $331(29.4)$ \\
\hline Erosions Present* & $100(9.52)$ \\
\hline Nodule present* & $13.10(1.44)$ \\
\hline Hb, mg/dl & \\
\hline & $24.00(12-41)$ \\
\hline ESR, Mm/hr (Median, IQR) & \\
\hline PROMS & $33.77(10.63)$ \\
\hline SF36BP & $46.34(11.15)$ \\
\hline SF36MH & $1.08(0.76)$ \\
\hline HAQ & $43.50(25.6)$ \\
\hline PGA & \\
\hline & \\
\hline & \\
\hline
\end{tabular}




\begin{tabular}{|c|c|}
\hline \multicolumn{2}{|l|}{ Medication/Comorbidity } \\
\hline Medication use* & $935(78.31)$ \\
\hline RDCl (Median, IQR) & $1.00(0-2)$ \\
\hline \multicolumn{2}{|l|}{ Others } \\
\hline Duration, Months (Median, IQR) & $12.00(7-18)$ \\
\hline $\begin{array}{l}\text { Baseline characteristics of } \\
\text { SF36VT - SF36 Vitality, Age } \\
\text { Patient's Global Assessmen } \\
\text { - Disease Activity Score (ESF } \\
\text { - Rheumatic Disease Comor } \\
\text { Joint Count, SJC - Swollen } \\
\text { Mass Index, PROMs -Patien } \\
\%)\end{array}$ & $\begin{array}{l}\text { (SD) unless otherwise stated. } \\
\text { essment Questionnaire, PGA- } \\
\text { bin Concentration, DAS28ESR } \\
\text { MH - SF36Mental health, RDCI } \\
\text { ration in Months, TJC - Tender } \\
\text { dimentation rate, BMI - Body } \\
\text { - Inter Quartile Range. }{ }^{*}=\mathrm{n} \text {, }\end{array}$ \\
\hline
\end{tabular}


Table 2: Multivariable predictors of vitality at baseline

\begin{tabular}{|c|c|c|c|}
\hline \multicolumn{4}{|c|}{ Multivariable Linear Regression $\left(n=973, R^{2}=0.49\right)$} \\
\hline Characteristic & Unit & Coefficient (Cl) & Standardised values \\
\hline Age & years & $0.01(-0.03$ to 0.05$)$ & 0.01 \\
\hline Sex & female & $-2.00(-3.11 \text { to }-0.86)^{*}$ & -0.08 \\
\hline TJC & $0-28$ & $-0.10(-0.19$ to -0.02$) *$ & -0.06 \\
\hline Pain & $0-100$ & $0.21(0.15$ to 0.28$) *$ & 0.24 \\
\hline Mental health & $0-100$ & $0.42(0.36$ to 0.47$) *$ & 0.42 \\
\hline HAQ & $0-3$ & $-2.43(-3.37$ to -1.50$) *$ & -0.17 \\
\hline
\end{tabular}


Table 3: Baseline Characteristics based on Trajectory groups

\section{Baseline}

\begin{tabular}{|c|c|c|c|}
\hline Characteristic & Unit & Group 1 (Fatigue $n=391$ ) & Group 2 (No -Fatigue $n=338$ ) \\
\hline \multicolumn{4}{|l|}{ Demographics } \\
\hline Age & years & $56.44(13.06)$ & $56.67(14.00)$ \\
\hline Sex & Female & $292(74.68)$ & $201(59.47)$ \\
\hline White Ethnicity & $Y$ & $384(98.21)$ & $325(96.15)$ \\
\hline BMI median, IQR & $\mathrm{Kg} / \mathrm{m} 2$ & $27.91(24.75-31.63)$ & $26.29(23.72-29.36)$ \\
\hline \multicolumn{4}{|l|}{ Smoking status (n, \%) } \\
\hline Never & & $137(36.05)$ & $143(43.73)$ \\
\hline Current & & $109(28.68)$ & $78(23.85)$ \\
\hline Ex-Smoker & & $134(35.26)$ & $106(32.42)$ \\
\hline \multicolumn{4}{|l|}{ Disease } \\
\hline \multicolumn{4}{|l|}{ Activity/Inflammation } \\
\hline DAS28ESR & & $5.14(1.47)$ & $4.39(1.53)$ \\
\hline TJC median, IQR & $0-28$ & $8(3-14)$ & $4(1-9)$ \\
\hline SJC median, IQR & $0-28$ & $5(2-10)$ & $4(1-9)$ \\
\hline Seropositive (n, \%) & Y & $211(63.94)$ & $178(61.38)$ \\
\hline Erosion (n, \%) & $Y$ & $113(30.87)$ & $100(32.36)$ \\
\hline Nodules (n, \%) & $Y$ & $36(10.11)$ & $28(9.21)$ \\
\hline $\mathrm{Hb}$ & $\mathrm{Mg} / \mathrm{dl}$ & $13.08(1.40)$ & $13.15(1.46)$ \\
\hline ESR & $\mathrm{Mm} / \mathrm{hr}$ & $27(13-47)$ & $20(12-36)$ \\
\hline \multicolumn{4}{|l|}{ PROMS } \\
\hline SF36BP & $0-100$ & $29.21(9.14)$ & $37.90(9.14)$ \\
\hline SF36VT & $0-100$ & $35.20(8.51)$ & 49.09 (8.39) \\
\hline SF36MH & $0-100$ & $42.33(10.84)$ & $52.02(9.15)$ \\
\hline HAQ & $0-3$ & $1.29(0.72)$ & $0.79(0.67)$ \\
\hline PGA & $0-100$ & $50.74(23.60)$ & $35.92(24.09)$ \\
\hline \multicolumn{4}{|l|}{ Comorbidity /Medication } \\
\hline $\mathrm{RDCl}$ & $0-9$ & $1(0-2)$ & $0(0-1)$ \\
\hline medication $(n, \%)$ & $Y$ & $318(81.33)$ & 291 (86.09) \\
\hline \multicolumn{4}{|l|}{ Others } \\
\hline Disease Duration median, IQR & months & $12(7-23)$ & $13(8-21)$ \\
\hline
\end{tabular}

SF36VT - SF36 Vitality, Age - Age at onset, HAQ - Health Assessment Questionnaire, PGA- Patient's Global Assessment of Disease Activity, $\mathrm{Hb}$ - haemoglobin Concentration, DAS28ESR - Disease Activity Score (ESR), SF36BP - SF36 Bodily Pain, SF36MH - SF36Mental health, RDCI - Rheumatic Disease Comorbidity Index, Duration - Disease Duration in Months, TJC - Tender Joint Count, SJC - Swollen Joint Count, ESR - Erythrocyte Sedimentation rate, BMI - Body Mass Index, IQR - Inter Quartile Range. 
Table 4: Baseline predictors of vitality group membership

\begin{tabular}{|c|c|c|c|}
\hline \multirow[t]{2}{*}{ Variable } & \multicolumn{2}{|c|}{ Univariate logistic regression } & \multirow{2}{*}{$\begin{array}{c}\text { Multiple logistic regression } \\
\text { OR }\end{array}$} \\
\hline & Unit & OR (Cl) & \\
\hline Age & Yrs. & 1.01 (0.99 to1.02) & 1.01 (0.99 to 1.02 ) \\
\hline BMI & $\mathrm{kg} / \mathrm{m}^{2}$ & $1.07 *(1.02$-to1.11) & $1.06 *(1.02$ to 1.10$)$ \\
\hline HAQ & $0-3$ & $1.57 *$ (1.09 to 2.27$)$ & $1.69 *(1.22$ to 2.33$)$ \\
\hline Mental health & $0-100$ & $0.94 *(0.92$ to 0.96 & $0.94 *(0.92$ to 0.96$)$ \\
\hline Bodily pain & $0-100$ & $0.95 *(0.93$ to 0.98$)$ & $0.96 *(0.93$ to 0.98$)$ \\
\hline Female & $Y$ & $1.71 *(1.08$ to 2.71$)$ & $1.94 *(1.28$ to 2.95$)$ \\
\hline $\begin{array}{l}\text { Comorbidities } \\
\text { (RDCl) }\end{array}$ & $0-9$ & $1.56 *$ (1.20 to 1.54$)$ & $1.32 *(1.12$ to 1.56$)$ \\
\hline PGA & $0-100$ & 1.00 (0.99 to 1.01$)$ & \\
\hline TJC & $0-28$ & 1.02 (0.99 to 1.06$)$ & \\
\hline SJC & $0-28$ & 1.02 (0.99 to 1.04$)$ & \\
\hline ESR & $(\mathrm{mm} / \mathrm{hr})$ & 0.99 (0.99 to 1.00$)$ & \\
\hline Medication & $\mathrm{y}$ & 0.83 (0.48 to 1.46$)$ & \\
\hline DAS28-ESR & & $1.40 *(1.25$ to 1.56$)$ & \\
\hline \multicolumn{4}{|c|}{$\begin{array}{l}\text { Univariate and multiple logistic regressions giving odds ratios (OR) with } 95 \% \text { confidence intervals }(\mathrm{Cl}) \text {. } \\
\text { Multiple logistic regression model included age, sex, and additional variables significantly predictive of } \\
\text { Vitality Group membership in univariate analysis, except that DAS28-ESR was excluded due to } \\
\text { substantial collinearity with component indices. }\end{array}$} \\
\hline \multicolumn{4}{|c|}{$\begin{array}{l}\text { Age, HAQ - Health Assessment Questionnaire, PGA- Patient's Global Assessment of Disease Activity, Hb } \\
\text { - haemoglobin Concentration, DAS28ESR - Disease Activity Score (ESR), RDCI - Rheumatic Disease } \\
\text { Comorbidity Index, TJC - Tender Joint Count, SJC - Swollen Joint Count, ESR - Erythrocyte Sedimentation } \\
\text { rate, BMI - Body Mass Index }\end{array}$} \\
\hline
\end{tabular}


Figure 1: Vitality trajectories over measurement occasions

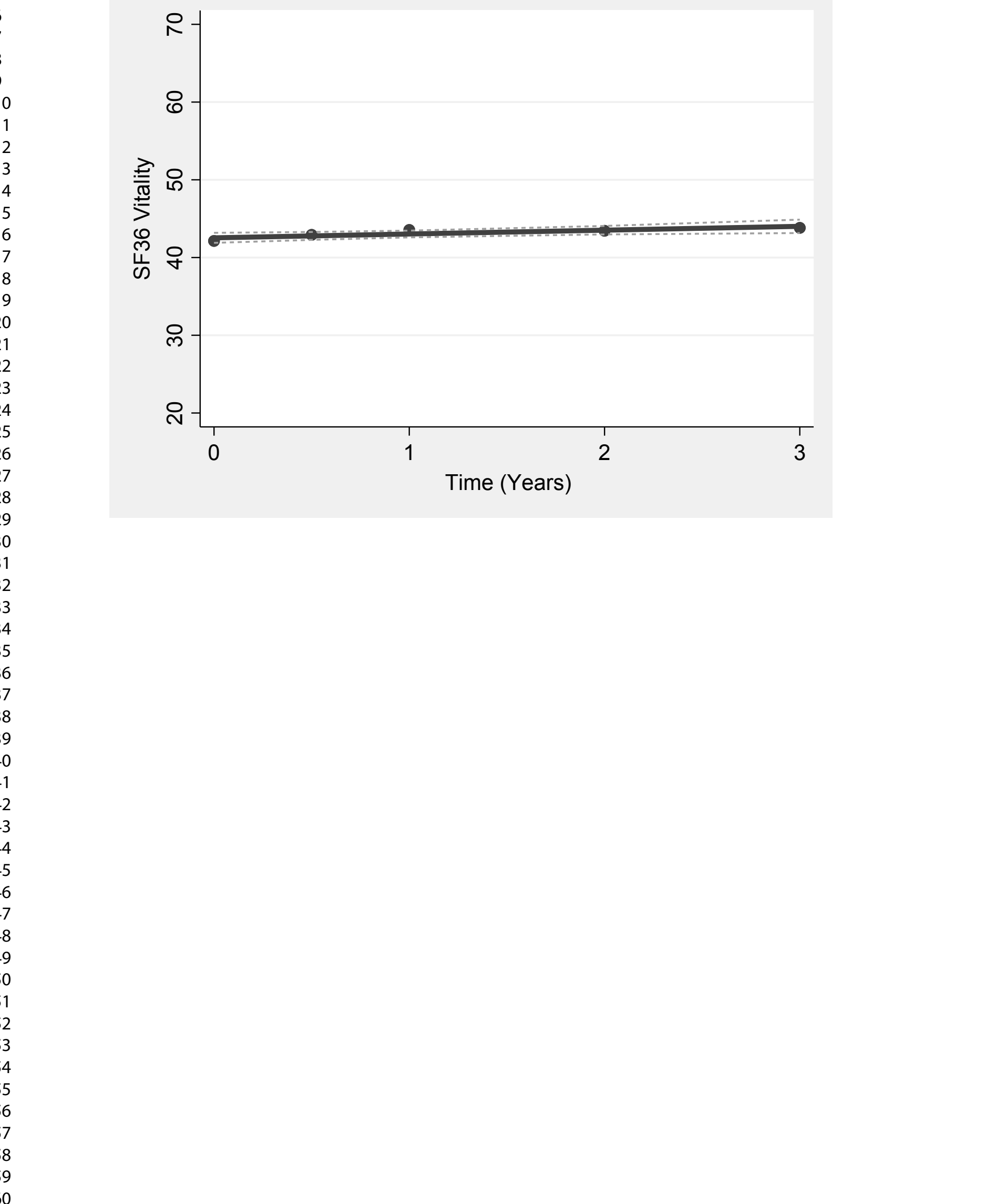


Figure 2: Vitality Trajectory groups based on GBTM analysis

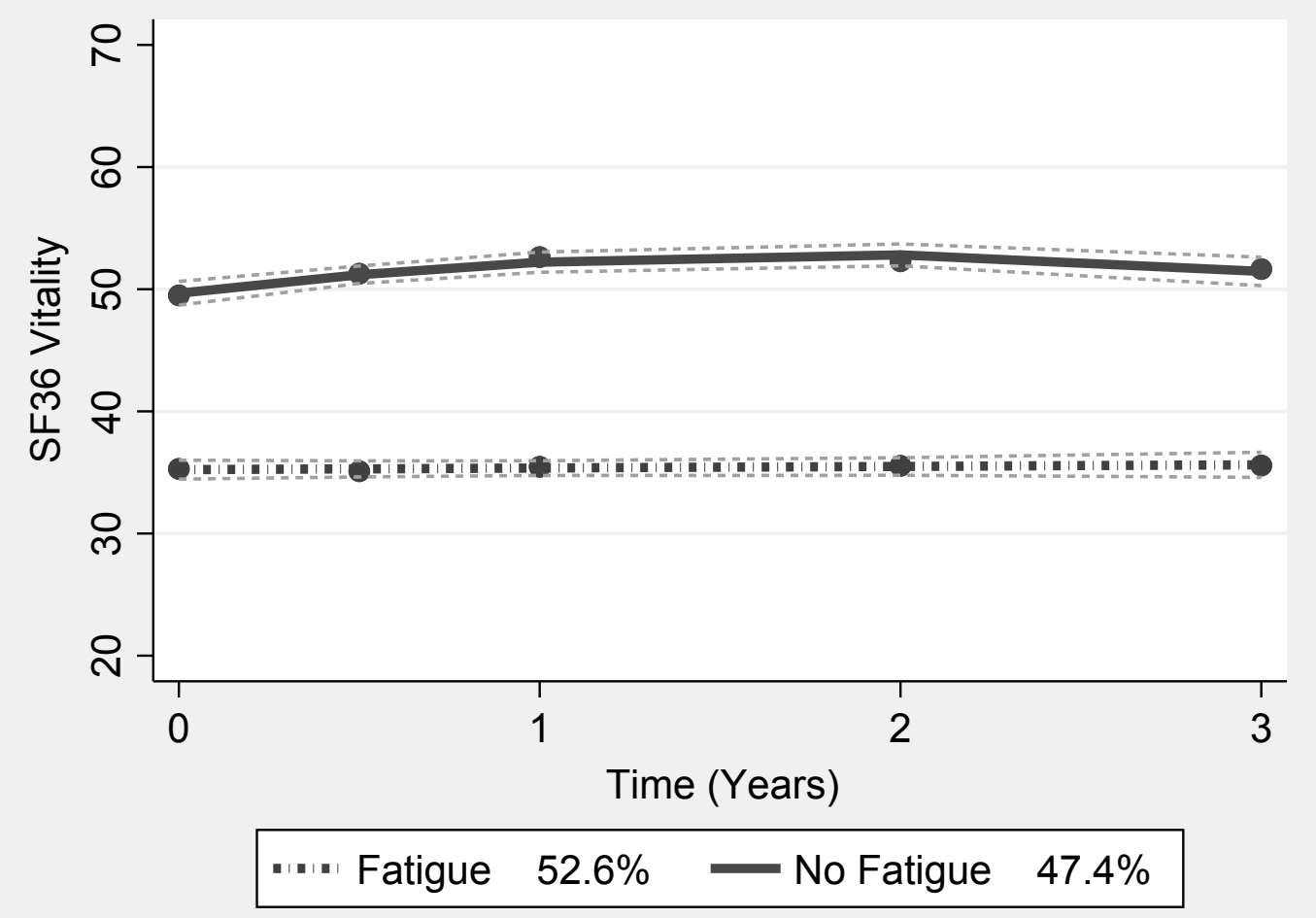

